\title{
Análisis del impacto de la política de atención a niños de 1 a 3 años, en el bienestar de sus familias en el caso del cantón Chunchi
}

\section{Analysis of the impact of the care policy for children from 1 to 3 years of age, on the well-being of their families in the case of the Chunchi.}

Quezada Vintimilla Vicky Paola. ${ }^{1}$, Rosales Namicela Mónica Briggith. ${ }^{2}$, \& Castillo Ortega Yonimiler. $^{3}$

\begin{abstract}
.
DOI: https://doi.org/10.33262/cienciadigital.v5i1.1520

Local development is the process of transformation of the local economy and society, it is related to well-being, growth, progress and sustainability. Local development is achieved through responsible productive development and the strengthening of society. Local development goes hand in hand with public policies that are instruments through which public authorities act, first by recognizing socially constructed problems within a community, and then, by incorporating, programming and executing actions aimed at their solution. The Child Development Centers within the framework of comprehensive protection, are inclusive and intercultural and provide their users with the services of: receptive care, health and nutrition, play and learning space, protective environments for a free expression of emotions. This research is of a mixed cross-sectional and descriptive type, the theoretical and empirical method (survey and interview) was applied. With the results obtained, it was possible to determine that the child development centers are complying with their commitment since they house children in a state of vulnerability and poverty, local development is generated because families can work and generate income for their homes, influencing this in the

\footnotetext{
${ }^{1}$ Universidad Católica de Cuenca, Posgrado, Maestría en Desarrollo Local Mención en Ordenamiento Territorial, Cuenca, Ecuador, vicky.quezada@est.ucacue.edu.ec. https://orcid.org/0000-0002-5903-8375

${ }^{2}$ Universidad Católica de Cuenca, Carrera de Economía, Cuenca Ecuador, mrosalesn@ucacue.edu.ec, https://orcid.org/0000-0002-3240-1146

3 Universidad Católica de Cuenca, Posgrado, Maestría en Desarrollo Local Mención en Ordenamiento Territorial, Cuenca, Ecuador, ycastilloo@ ucacue.edu.ec , https://orcid.org/0000-0002-7710-5199
} 
reduction of gender violence. We can conclude that $100 \%$ of users feel well when they send their children to this Children's Center.

Keywords: Local development, public policies, child centers

\section{Resumen.}

Introducción: El desarrollo local es el proceso de transformación de la economía y de la sociedad local, está relacionado con el bienestar, el crecimiento, el progreso y la sostenibilidad. El desarrollo local se logra mediante un fomento productivo responsable y el fortalecimiento de la sociedad; va de la mano con las políticas públicas que son instrumentos mediante los cuales las autoridades públicas actúan, primero reconociendo problemas socialmente construidos en el seno de una comunidad, y luego, mediante la incorporación, programación y ejecución de acciones dirigidas a su solución. Los Centros de Desarrollo Infantil en el marco de la protección integral, son inclusivos e interculturales y aportan a sus usuarios los servicios de: atención receptiva, salud y nutrición, espacio de juego y aprendizaje, entornos protectores para una libre expresión de emociones. Metodología: Esta investigación es de tipo mixta de corte trasversal y descriptiva, se aplicó el método teórico y empírico (encuesta y entrevista). Resultados: se pudo determinar que los centros de desarrollo infantil están cumpliendo con su acometido ya que albergan a niños en estado de vulneración y pobreza, se genera desarrollo local porque las familias pueden laborar y generar ingresos para sus hogares, incidiendo esto en la reducción de la violencia de género. Conclusión: el 100\% de los usuarios siente bienestar al enviar a sus hijos a estos centros Infantiles.

Palabras claves: Desarrollo Local, política publica, Centros de Desarrollo Infantil (CDI), Chunchi

\section{Introducción.}

El desarrollo local es un proceso de trasformación de la economía y de la sociedad local, busca mejorar las condiciones de vida de su población mediante una acción decidida y consensuada entre los diferentes agentes socioeconómicos locales, públicos y privados. El objetivo principal es construir las capacidades institucionales y productivas de un determinado territorio, a fin de mejorar su futuro económico y la calidad de vida de sus habitantes (Díaz Jesús, 2018). El desarrollo local se logra mediante un fomento productivo responsable y el fortalecimiento de la sociedad (Mauricio et al., 2003).

Para lograr el desarrollo local, las políticas públicas son herramientas mediante las cuales, las autoridades públicas actúan reconociendo problemas socialmente generados en el seno de una comunidad, que son impulsadas por un gobierno para respetar, proteger y velar por los derechos de las personas, de forma individual y colectiva (Charry \& Pérez, 2014). 
Un instrumento importante que acoge las políticas públicas de las naciones y que fue trabajada y aprobada en el 2015, es la Agenda 2030 y los 17 Objetivos de Desarrollo Sostenible (ODS); 192 países acogen dicha herramienta para la efectiva implementación de esta hoja de ruta hacia la dignidad, la justicia y la equidad en el planeta. El 19 de abril de 2018, el presidente de la República del Ecuador, Lenín Moreno, mediante Decreto Ejecutivo 371, reitera el compromiso y declara como política pública del Gobierno Nacional la adopción de la Agenda 2030 para el Desarrollo Sostenible (Secretaría Técnica Planifica Ecuador, 2019).

Las políticas y programas para la primera infancia declaradas por el actual gobierno, tienen en cuenta las principales necesidades y los derechos de los niños y las familias. El objetivo es proporcionar servicios, programas y atenciones que permitan que los niños crezcan y se desarrollen a nivel cognitivo, socioemocional y físico, mientras que se garanticen todos sus derechos incluyendo oportunidades para su cuidado, educación y protección (European Environment Agency (EEA), 2019).

La vulneración de derechos contra niños, niñas y adolescentes, constituye un serio problema a nivel global, ante el cual la política pública aún enfrenta el desafío de articular una respuesta eficaz que responda a una perspectiva de derechos a favor de los grupos vulnerables (Contreras et al., 2015). En este escenario es imperativo desarrollar y fortalecer las políticas públicas de protección social y las del ámbito del mercado laboral en igualdad de condición (CEPAL, 2018).

"El Ecuador pretende generar políticas integrales y metas que permitan monitorear el cierre de brechas y el ejercicio pleno de los derechos, entendiendo como un reto a superar la incorporación de la ciudadanía en el seguimiento y evaluación de las políticas públicas" (SENPLADES, 2017, p. 34). Una de las políticas públicas con mayor relevancia e importancia es velar por los derechos de los niños; por ello se considera fundamental la creación de centros de desarrollo infantil, cuyo objetivo es la protección de niños en su primera infancia (Ministerio de Inclusión Económica y Social, 2019).

Como parte de la implementación de políticas públicas sociales orientas a la protección de los derechos de los niños de 1 a 3 años, en el cantón Chunchi se crearon tres centros de Desarrollo Infantil en tres diferentes sectores: Chunchi, Gonzol y Llagos dotados de capital humano calificado, recursos económicos e infraestructura física ajustada a las normas técnicas establecidas por el órgano rector, el Ministerio de Inclusión Económica y Social MIES. Estas políticas públicas tienen un enfoque social y buscan mejorar la calidad de vida tanto de los niños como de sus familias.

El cantón Chunchi está ubicado en la zona Sur de la provincia de Chimborazo, tiene una superficie total de 274,9 km2. Está compuesto por 5 parroquias, de las cuales una es urbana: Chunchi Matriz, y cuatro son rurales: Gonzol, Capzol, Llagos y Compud. La distribución es 
concentrada porque no existe dispersión mayor entre los centros poblados y la parroquia Matriz. La población del cantón Chunchi es de 12.795 habitantes, entre ellos 979 niños entre las edades de 1 a 4 años según las proyecciones (INEC, 2020).

Instituciones como el MIES y el Gobierno autónomo descentralizado del cantón Chunchi, mediante convenio proveen a los centros de desarrollo infantil: alimentación, educación, trasporte, salud y materiales lúdicos, para que la jordana diaria de los usuarios sea completa y placentera. Todo lo antes mencionado es un servicio de atención gratuita y enfocada en ayudar a los sectores más vulnerables del cantón. Pese a estas normativas técnicas, se ha observado durante años la falta de ocupación de estos servicios por parte de las familias del cantón Chunchi y sus alrededores, existe una falta de cobertura; por falta de información, falta de educación, ruralidad u otros factores.

En Chunchi existen familias de bajos recursos económicos de acuerdo con el Sistema Integrado de Indicadores Sociales del Ecuador (SIISE), la pobreza por necesidades básicas insatisfechas alcanza el $75,13 \%$ de la población total del cantón, y la pobreza extrema el $54,94 \%$, estos porcentajes son elevados y representan la vulnerabilidad de las familias y niveles elevados de desnutrición crónica 64.4\% según el INEC 2010 (GAD Municipal Chunchi, 2014). Las familias del cantón Chunchi, generalmente las madres se ven obligadas a trabajar con los niños en brazos, o descuidando a sus hijos mientras desarrollan largas jornadas de trabajo, sin proveerle de alimento durante el día. En otros casos las madres no pueden laborar por cumplir el papel de ama de casa, de esta manera no provee recursos a su hogar, muchas veces quedando rezagadas y sin cumplir sus metas y objetivos.

\section{Gráfico 1}

Mapa de Chunchi y ubicación de los CDI

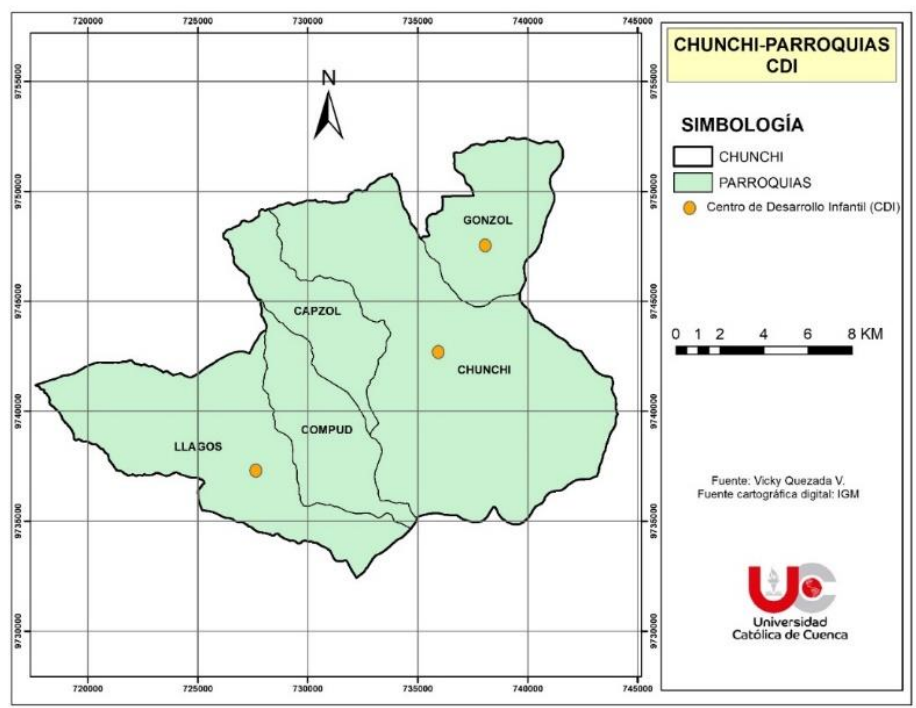

Fuente: Elaboración propia. 
Por otra parte, la pandemia del momento "El coronavirus (COVID-19)" ya se ha extendido a los 36 países y territorios de América Latina y el Caribe. Esta pandemia es tanto una crisis de salud como una amenaza social y económica, creando situaciones desafiantes, particularmente para los más vulnerables. El impacto en los niños, niñas, adolescentes y sus familias será devastador, con enfermedades, aislamiento, miedo y pérdida de medios de vida. Se estima que el número de personas en situación de pobreza podría aumentar de 185 a 220 millones de personas; mientras que el número de las personas en extrema pobreza podrían aumentar de 67,4 a 90 millones (UNICEF, 2020).

A raíz de esta problemática se plantea la presente investigación que busca atender ¿Cuál es el impacto de la política pública en la creación de estos centros?, y para poder responder a esta interrogante el objetivo principal de la investigación es diseñar una metodología que mida el impacto de la política de atención a niños de 1 a 3 años, en el bienestar de sus familias en el caso del cantón Chunchi.

\section{Fundamentación teórica.}

\section{Desarrollo Local}

El desarrollo local tiene sus orígenes en la expansión de procesos económicos y está relacionado con otros conceptos, como son: el bienestar, el crecimiento, el progreso y la sostenibilidad. El desarrollo, no solo hace referencia al crecimiento económico, sino también a un crecimiento social y cultural en un sentido más amplio e integrado. Se puede definir el desarrollo local como el proceso de transformación de la economía y de la sociedad local, que busca mejorar las condiciones de vida de su población (Díaz, 2018). Para el desarrollo local hace falta una visión del proceso económico, entendido como la transformación de los recursos naturales en bienes y servicios para la satisfacción de las necesidades humanas. La sostenibilidad de ese proceso va ligada a la sostenibilidad de la base de recursos de la que se alimenta (Falconí, 2017). Un primer desafío pasa por recuperar el vínculo gobiernociudadano, elemento clave para la legitimidad de las formas de gobierno (Méndez, M. 2016).

(Gallicchio, 2017) Manifiesta el desarrollo local mejora la gobernanza en todos sus niveles, ya que sitúa al gobierno y al poder, al alcance de la ciudadanía. El desarrollo local se logra mediante un fomento productivo responsable y el fortalecimiento de la sociedad. A estos elementos, hay que sumar los aspectos ambientales para alcanzar un desarrollo sostenible (Mauricio et al., 2003).

Con el fin de dar respuesta a las diferentes problemáticas que se presentan en un Estado, los gobernantes se ven en la obligación de implementar una serie de estrategias y actividades, a través de la creación de políticas públicas (Pino Montoya, 2017). Es así que para que exista desarrollo local deben implementarse políticas en bienestar de la sociedad. 


\section{Políticas públicas}

Las políticas públicas son determinadas configuraciones de los actores, que se construyen para obtener un bien común, es necesario entender la diversidad y diferencia entre organizaciones gubernamentales, públicas y organizaciones no gubernamentales, por mencionar unas cuantas, y sus implicaciones en la formulación, implementación, ejecución y evaluación de políticas públicas (Charry \& Pérez, 2014).

Las políticas públicas son instrumentos mediante los cuales las autoridades públicas actúan, primero reconociendo problemas socialmente construidos en el seno de una comunidad, y luego, mediante la incorporación, programación y ejecución de acciones dirigidas a su solución o manejo. Las políticas públicas son patrones que impulsa un gobierno para respetar, proteger y realizar los derechos de las personas, de forma individual y colectiva, garantizando todos los derechos y el buen vivir de los ciudadanos. (Charry \& Pérez 2014).

Las políticas para la primera infancia, son políticas públicas que proporcionan visiones y objetivos a nivel nacional para movilizar acciones que garanticen los derechos de los niños y proporcionen los servicios y programas necesarios para asegurar su bienestar y desarrollo. El desarrollo infantil es fundamental para alcanzar las metas de desarrollo sostenible y la garantía de los derechos durante los primeros años de vida, uno de los pilares fundamentales de los derechos humanos (European Environment Agency (EEA), 2019).

Muller (2010) plantea que la política pública busca atender conflictos; su actividad y campo de acción se da cuando una serie de actores percibe que su situación no es conforme a la que debería ser o podría ser. Las políticas públicas no son solamente un proceso de decisión, sino el lugar donde una sociedad construye su relación con el mundo. Entonces una política pública, es la construcción de una imagen de la realidad sobre la cual se quiere intervenir, con el propósito y objetivo de brindar servicios a quien más lo necesita (Charry \& Pérez, 2014). En la actualidad, la participación ciudadana se ha convertido en una estrategia imprescindible para la legitimación de políticas públicas, sin embargo esto no supone la implementación de instancias realmente democráticas de involucramiento de la ciudadanía (Guardamagna \& Reyes 2019).

La formulación de la política pública tiene un proceso previo a la formalidad de las aprobaciones en las instancias gubernamentales; parten de la evaluación diagnóstica de la política, la institucionalidad y los servicios, posterior a esto se procede a la elaboración de propuestas que identifiquen los aspectos críticos donde se va a intervenir; la planificación estratégica del sector y de los actores involucrados, el coste de la implementación de la política y finalmente, la programación de una línea de tiempo hasta llegar a la declaratoria pública y su ulterior implementación (Ministerio de Inclusión Económica y Social, 2013).

En el Ecuador, una de las políticas públicas con mayor relevancia e importancia es velar por los niños, por este motivo el Gobierno Nacional ha creado centros de desarrollo infantil, 
cuyo objetivo es la protección de niños en su primera infancia (Ministerio de Inclusión Económica y Social, 2019).

\section{Centros de Desarrollo infantil y los impactos en las familias}

Los servicios de cuidado infantil para niños menores de cuatro años de edad han expandido su cobertura de forma importante en América Latina y el Caribe (ALC). La mayoría de los programas públicos que proveen servicios de cuidado infantil en ALC fueron creados como una respuesta a la creciente participación laboral de las mujeres. Se pensó que estos servicios podrían facilitar el empleo de las madres, sobre todo de aquellas pertenecientes a estratos socioeconómicos bajos, no solamente las mujeres participan con mayor frecuencia en los mercados laborales de la región, sino que además contribuyen con una fracción importante de los ingresos que sustentan a sus familias, el trabajo ha dignificado a las mujeres, ya no se encuentran sometidas por no poder colaborar económicamente en el hogar, de esta manera se reduce el maltrato hacia las mujeres (Araujo \& López, 2015). Las desigualdades del desarrollo humano dañan las sociedades y debilitan la cohesión social y la confianza de la población en los gobiernos, las instituciones y sus congéneres (PNUD, 2019).

Para el desarrollo Infantil integral, la familia es el grupo fundamental de la sociedad, una institución que ha compartido siempre las mismas funciones entre ellas: la crianza de los hijos, la supervivencia y la unión entre sus miembros; la familia se ha considerado una fuente primaria de apoyo social (Hombrados, M. \& Olmos, C, 2016).

Las Naciones Unidas ONU (2015) afirma que durante la última generación, cientos de millones de personas han salido de la pobreza extrema, existe un aumento considerable de niños y niñas que tienen acceso a la educación. La Asamblea General (Naciones Unidas, 2016) manifiesta que uno de sus principales objetivos es asegurar que nadie se quede atrás, lo que implica poner fin a la pobreza incluida: la erradicación de la extrema pobreza; garantizar la igualdad de derechos y oportunidades; la diversidad socioeconómica, cultural y la integración en el espacio urbano, mejorar la habitabilidad la educación, la seguridad alimentaria y la nutrición, la salud y el bienestar.

Dentro de la agenda 2030 y de sus Objetivos de Desarrollo sostenible (ODS), encontramos que los tres primeros objetivos, justifican la creación de los centros de desarrollo infantil; el primer objetivo: fin de la pobre, erradicar la pobreza en todas sus formas; el segundo objetivo: hambre cero, busca terminar con todas las formas de hambre y desnutrición; y finalmente el tercer objetivo: salud y bienestar, es fundamental garantizar una vida saludable y promover el bienestar para todos a cualquier edad. Se han obtenido grandes progresos en relación con el aumento de la esperanza de vida y la reducción de algunas de las causas de muerte más comunes relacionadas con la mortalidad infantil y materna (CEPAL, 2019).

Los Centros de Desarrollo Infantil en el marco de la protección integral, son inclusivos e interculturales y aportan a sus usuarios los servicios de: atención 
receptiva, salud y nutrición, espacio de juego y aprendizaje, entornos protectores para una libre expresión de emociones. La atención integral se orienta a través de la Ruta Integral de Atenciones que organiza y articula la intersectorialidad con el Ministerio de Salud Pública (MSP), Ministerio de Educación, Gobiernos Autónomos Descentralizados (GAD), Registro Civil, Ministerio de Agricultura y Ganadería, Organizaciones de Sociedad Civil entre otros actores (Ministerio de Inclusión Económica y Social, 2019, p. 32).

El Ministerio de Inclusión Económica y Social (2013) manifiesta que la atención en los primeros años de vida, desde la gestación, nacimiento hasta el crecimiento, es una de las prioridades para el desarrollo pleno del ser humano. Mediante los siguientes argumentos justifica la construcción de la política pública: el primer argumento son los beneficios que ofrece la inversión en el Desarrollo Infantil Integral; otro argumento es de tipo científico, el cerebro que posee un niño dentro de los seis primeros años, es el que se tendrá para toda la vida, esto hace del período inicial de vida una etapa determinante para el Desarrollo Integral del individuo.

En el Ecuador la desnutrición es un estado patológico resultante de una dieta deficiente en uno o varios nutrientes esenciales o de una mala asimilación de los alimentos, prevalencias comparativas de desnutrición crónica, en menores de dos años; según la Encuesta Nacional de Salud y Nutrición ENSANUT (2018), registra que el 27.2\% de niños tienen desnutrición crónica en Ecuador (INEC, 2020). Esto ocurre en su gran mayoría porque las madres se encuentran laborando y los niños no tienen una ingesta adecuada de alimentos, o por falta de recursos económicos.

Los centros de Desarrollo Infantil, no solo ayudan a que el menor crezca sano físicamente, también su desarrollo psicológico es muy importante, ver que su familia se desenvuelve en un ambiente de paz y calma ayuda a que el niño en desarrollo no tenga traumas de su infancia, por maltrato familiar o infantil. En el Ecuador a lo largo de la vida 65 de cada 100 mujeres, han experimentado por lo menos un hecho de algún tipo de violencia en alguno de los distintos ámbitos. En los últimos 12 meses, 32 de cada 100 mujeres en el Ecuador han experimentado algún tipo de violencia según la Encuesta Nacional sobre Relaciones familiares y violencia de Género en contra de las mujeres ENVIGMU (INEC, 2019).

Finalmente hablar de centros de desarrollo infantil es "sembrar para el futuro", es decir que niños y niñas están siendo criados en base a estimulación, cuidado y protección, todo esto guiado y regido mediante leyes que amparan la creación de esta política pública.

\section{Políticas en relación a la protección infantil y familiar y la institución que vela por el cumplimiento de este derecho}

La constitución de la República del Ecuador (Asamble Nacional, 2008) contempla varios artículos que atienden está problemática; por mencionar: En el Art. 44.- El Estado, la 
sociedad y la familia promoverán de forma prioritaria el desarrollo integral de las niñas, niños y adolescentes, y asegurarán el ejercicio pleno de sus derechos. Las niñas, niños y adolescentes tendrán derecho a su desarrollo integral, entendido como proceso de crecimiento, maduración y despliegue de su intelecto y de sus capacidades, potencialidades y aspiraciones, en un entorno familiar, escolar, social y comunitario de afectividad y seguridad.

En el Art. 45.- Las niñas, niños y adolescentes gozarán de los derechos comunes del ser humano, además de los específicos de su edad. El Estado reconocerá y garantizará la vida, incluido el cuidado y protección desde la concepción.

En relación a los artículos antes mencionados en el Art. 46.- El Estado adoptará las medidas que aseguren a las y los menores de seis años una atención que garantice su nutrición, salud, educación y cuidado diario en un marco de protección integral de sus derechos.

En el Código de la Niñez y Adolescencia (2013), algunos artículos atienda de manera directa esta temática así: El Art. 11.- El interés superior del niño es un principio que está orientado a satisfacer el ejercicio efectivo del conjunto de los derechos de los niños, niñas y adolescentes; e impone a todas las autoridades administrativas y judiciales y a las instituciones públicas y privadas, el deber de ajustar sus decisiones y acciones para su cumplimiento.

Art. 12.- Prioridad absoluta. - En la formulación y ejecución de las políticas públicas y en la provisión de recursos, debe asignarse prioridad absoluta a la niñez y adolescencia, a las que se asegurará, además, el acceso preferente a los servicios públicos y a cualquier clase de atención que requieran.

El MIES mediante el Acuerdo Ministerial $N^{\circ} 006$ de 22 de enero de 2018 expide las directrices para la prevención y atención de la violencia física, sicológica y sexual detectada en los servicios de atención del Ministerio de Inclusión Económica y Social en contra de niñas, niños, adolescentes, personas con discapacidad y personas adultas mayores (2019).

En el Código Orgánico de Organización Territorial, Autonomía y Descentralización (COOTAD, 2010) se establece la coordinación multinivel. Adicionalmente, incluye en su artículo 54, las funciones del gobierno autónomo descentralizado municipal relacionadas con el ejercicio de los derechos de la niñez y adolescencia.

La atención y cuidado diario de niñas y niños de 0 a 3 años fortalece su desarrollo a través de la libre expresión, incorporando actividades lúdicas y recreativas que promuevan experiencias de aprendizaje significativo y con pertinencia socio cultural; garantizando las atenciones descritas en la Ruta Integral de Atenciones (RIA). 
Para concluir este trabajo investigativo, podemos palpar que la creación de políticas públicas para el desarrollo local es de suma importancia, porque sea cual sea el enfoque que se lo dé, deben estar valorizadas y evaluadas a fin de cumplir objetivos de mejora social.

La evaluación de políticas públicas está basada en la investigación, de carácter interdisciplinario, es un proceso sistémico de observación, medida, análisis e interpretación que busca el conocimiento de una acción, para alcanzar un juicio valorativo fundado en evidencias en relación con su diseño, puesta en práctica, efectos, resultados e impactos mediante la aplicación de un método sistematizado con el fin de medir (resultados e impactos) (Urbanos, 2012). Pues bien, la política pública es una hipótesis, por tanto, la evaluación de la política es la comprobación de la hipótesis, un aspecto central en el desarrollo de la disciplina tiene como centro explicativo las relaciones causa-efecto. La evaluación es la fase menos desarrollada de la política pública, debido a las características técnicas constitutivas (Aguilar, 2017).

Villaroel et al. (2018) definen que evaluar las políticas públicas permite optimizar recursos con base en evidencia concreta. Tanto en la planificación como en la ejecución de un proyecto, la evaluación facilita la generación de intervenciones con mayores grados de eficiencia y eficacia. Como menciona Banks (2009) en el campo de las políticas públicas, lo que debe interesarnos es lo que de verdad funciona. Generar evidencia toma tiempo, y requiere de metodologías y procesos rigurosos, así como equipos técnicos capacitados; los resultados obtenidos serán óptimos y la utilización de recursos será eficaz.

Cardozo 2006 desarrolla una tipología de evaluación entre ellas las más importantes para este tema investigativo son:

\begin{tabular}{ll}
\hline \multicolumn{1}{c}{ Tipo de evaluación } & \multicolumn{1}{c}{ En que consiste } \\
\hline De eficacia & Verifica si los resultados han permitido lograr los objetivos. \\
De metas & Analiza lo logrado en comparación con lo planeado. \\
De eficiencia & $\begin{array}{l}\text { Relaciona los resultados con los esfuerzos realizados para lograrlos (costo- } \\
\text { beneficio, costo-eficacia). }\end{array}$ \\
de efectos & Mide las consecuencias de los resultados. \\
de impacto & $\begin{array}{l}\text { Se refiere al impacto global, provocado por la combinación de efectos } \\
\text { atribuibles al programa, sobre la problemática que justifica la existencia de la } \\
\text { actividad. }\end{array}$ \\
De satisfacción & $\begin{array}{l}\text { Determina el grado en que la población considera satisfecha su necesidad o } \\
\text { resuelto su problema. }\end{array}$ \\
\hline
\end{tabular}

Tabla 1: Tipos de evaluación

Fuente: Elaboración propia con base a Cardozo (Aguilar, 2017). 
Existe también la evaluación de la teoría del programa que tiene como finalidad comprender las cadenas o secuencias causales que provocan los resultados. Su intención es saber porque han ocurrido las cosas, cuando conocen el porqué es cuando se puede mejorar, proponiendo recomendaciones enfocadas y concretas que atañen a la totalidad de intervenciones. Por otra parte, está la evaluación orientada por criterios, un criterio es una definición sobre la situación deseable u optima de un proyecto, funciona sometiendo a examen al programa y valorándolo con relación a ciertos criterios establecidos. Si alcanza los estándares establecidos se considera logrado el criterio (Banco de Desarrollo de América Latina, 2016).

\section{Fases de la evaluación orientada por criterios}

1. Construcción, reconstrucción o identificación de la teoría o modelo lógico

1.1. Resultados

1.2. Dimensión de procesos o mecanismos de implementación

1.3. Dimensión estructural: los recursos, la organización y otros elementos estructurales

1.4. La perspectiva sistémica

2. Múltiples perspectivas sobre las teorías que subyacen a una intervención

2.1 Fuentes para obtener las pistas y sugerencias para construir la teoría.

3. Modelo lógico con perspectiva sistémica

4. Graficar la teoría

5. Determinación de los aspectos a evaluar

6. Métodos y técnicas

7. Análisis

Tabla 2: Fases de evaluación

Fuente: Elaboración propia con base a (Banco de Desarrollo de América Latina, 2016).

El considerar una acción en beneficio de la población más vulnerable por parte de la política pública a través de centros de desarrollo infantil, es fundamental en la lucha contra la pobreza, debido a que mejora el desarrollo psicomotriz, social y emocional de los niños de 1 a 3 años, también permite a la mujer apoyar a la economía del hogar dedicándose a otras actividades y empoderándola para romper esquema de maltrato que se dan de manera importante en los hogares que se encuentran en condiciones de pobreza y extrema pobreza.

\section{Metodologia.}

El presente trabajo de investigación, es de tipo mixta de corte transversal y descriptiva.

Es cualitativa porque se analizan datos de condiciones socioeconómicas de los beneficiarios de los CDI y percepciones de los beneficios o bondades que prestan dichos centros de desarrollo infantil; será de tipo cuantitativa porque se hará un diagnóstico de las características económicas y sociales de quienes hacen uso de estos centros y la medición de parámetros de los servicios que prestan estos CDI, se aplicará un enfoque no experimental 
de corte transversal y el uso de la estadística descriptiva para medir el alcance de los centros en el área de influencia del cantón.

La investigación científica se basó en la aplicación de dos métodos: en un primer momento el método teórico, que permitió el análisis de teorías sobre evaluación de impacto de política pública, desarrollo local y centros de desarrollo infantil, mediante la técnica de revisión de bases de datos científicas y fuentes oficiales, con el fin de conocer que factores son necesarios de analizar como determinantes del impacto de este tipo de políticas para el desarrollo local.

Para la etapa del diagnóstico se aplicó el método empírico, mediante la técnica de la encuesta, esta herramienta permite conocer cuales es la condición socioeconómica de los beneficiarios del cantón y conocer cómo perciben los usuarios del servicio sobre la calidad de los CDI y los beneficios que les ha aportado a sus familias y su economía, llevando a sus niños de 1 a 3 años a estos centros.

El cantón Chunchi cuenta con tres centros uno en la cabecera cantonal con 40 familias beneficiarias, otro en Llagos con 27 beneficiarios y finalmente en Gonzol con 27 beneficiarios, estos datos fueron considerados al 14 de agosto del 2020 (fuente coordinadoras CDI), lo que indica que la población total es de 94 usuarios. Para la validación del instrumento se aplicó una encuesta piloto a seis representantes de los usuarios seleccionados al azar y posteriormente una vez validada la encuesta, se aplicó el instrumento a la totalidad de beneficiarios en un número de 94 .

La encuesta está compuesta por dos secciones, la primera que recaba información importante compuesta por 19 preguntas de cómo perciben los usuarios el servicio de los CDI (datos informativos), y una segunda sección compuesta por 11 preguntas que corresponden a datos personales. Toda la información recolectada nos ayudó a cumplir con el objetivo de nuestra investigación.

Con los datos obtenidos se procedió a elaborar una base de datos la misma que fue tabulada por medio del programa estadístico SPSS. Se utilizó estadística descriptiva para la elaboración de tablas y gráficas con sus análisis respectivos.

Además, para reforzar la información obtenida mediante la encuesta, se llevó a cabo entrevistas semiestructuradas a funcionarios vinculados al desarrollo de estos centro: el Ingeniero Jorge Luis Ortega Vicealcalde del cantón Chunchi, y la ingeniera Paulina Moreano Coordinadora distrital de Misión Ternura MIES; el objetivo de la entrevista fue indagar temas sobre recursos económicos, infraestructura, cobertura e impacto de estos centros en la calidad de vida de poblaciones vulnerables del cantón.

Finalmente, con los resultados obtenidos para en análisis del impacto de la política pública se aplicó el método inductivo; haciendo uso de la teoría propuesta por Aguilar 2017 y el 
Banco de Desarrollo de América Latina estas teorías nos permiten hacer un análisis detallado sobre el impacto que representa una política pública para el desarrollo local.

\section{Resultados y discusión.}

En este apartado se presenta el análisis de datos obtenidos de la investigación, los mismos que fueros tabulados y analizados mediante la estadística descriptiva. Se presentan los resultados más destacados que permite medir el real impacto que tienen los CDI en el desarrollo local del cantón de estudio.

Dentro de la encuesta planteada a los usuarios se obtuvo los siguientes resultados:

- En lo referente a características de los usuarios de los centros se puede concluir que: $27.66 \%$ tienen edades entre $12-24$ meses, $28.73 \%$ edades de 25 a 36 meses y el $43.62 \%$ de usuarios tienen más de 36 meses.

- La frecuencia con la que asisten los niños a los centros es: el $83.0 \%$ de usuarios asisten al CDI de manera regular, mientras que un $12.8 \%$ casi siempre, el $3.2 \%$ rara vez y el $1.1 \%$ algunas veces.

- Ademas de la informacion recolectada. Los representantes califican de muy bueno el $61.70 \%$, bueno el $37.23 \%$ en cuanto al desempeño fisico, psicologico, emocional y psicomotriz que muestras sus representados a raiz que asiste a los CDI.

- Los usuarios conocen totalmente los beneficios que prestan los CDI (61.7\%), medianamente $33 \%$, poco 5.3\%; sabiendo que en los centros de desarrollo infantil los niños reciben atención receptiva, salud y nutrición, espacios de juego y aprendizaje, entornos protectores para una libre expresión de emociones. Un porcentaje poco significativo desconoce los servicios que brindan los CDI.

- El 97.87\% de los representantes conocen que sus hijos tienen en los CDI, derecho a recibir: alimentación, cuidado, salud, atención de manera gratuita, sin ser maltratados ni violentados.

De la información recolectada el 96.8\% de los encuestados creen que se debería incorporar otro tipo de programas. Consideran que se debería implementar programas tales como: el 26.6\% Alimentación sana, aprender cuáles son los alimentos que los niños deben consumir y su forma de preparación, con el objetivo de brindar una alimentación de calidad; $25.5 \%$ consejería matrimonial, aprender a convivir con su pareja de manera armónica, y así evitar maltrato familiar o violencia de género; $41.5 \%$ talleres de aprendizaje, con el objetivo de aprender oficios los mismos que les permita insertarse en el campo laboral, así obtener ingresos y mejorar su condición de vida; $3.2 \%$ alcohólicos anónimos, en algunos casos donde sus esposos consumen alcohol de manera excesiva.

Existe una percepción positiva respecto a la atención que brinda el personal a los niños que acogen los CDI. El $78.27 \%$ califica de muy buena, el $20.21 \%$ califica de buena y el $1.06 \%$ califica de regular. 
La atención en el área de salud que reciben los niños por parte del Ministerio de Salud Pública indica que un $57.45 \%$ califica el servicio de salud de buena calidad, el $32.98 \%$ de muy buena, el $7.45 \%$ califica de regular y el $2.13 \%$ de mala.

Del análisis realizado en cuanto a la infraestructura de los CDI, un $67.02 \%$ de usuarios considera que está en buenas condiciones, el $18.09 \%$ en muy buena y el $14.89 \%$ regular. Sin embargo, un $88.11 \%$ piensa que se debería adecuar espacios lúdicos y recreativos para los niños con juegos infantiles plásticos para evitar accidentes.

¿Cómo considera usted que el CDI le ha ayudado a su familia?

Según los datos obtenidos el $100 \%$ de usuarios se ven beneficiados y pueden desarrollar otras actividades que generen bienestar para las familias como lo muestra la figura 6. Entre ellas un $84.78 \%$ de encuestados indica que ha podido incorporarse a la vida laboral y así generar ingresos económicos para su hogar mejorando su calidad de vida.

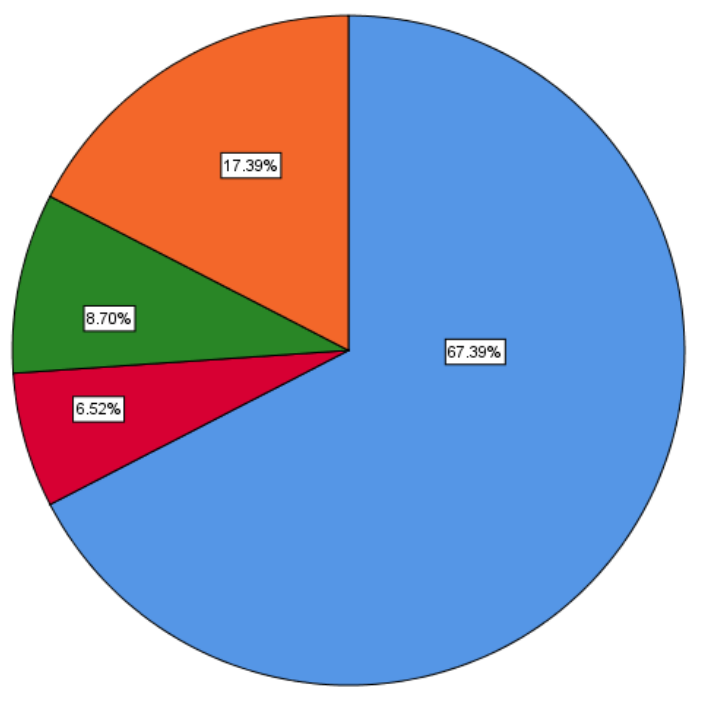

Figura 1. Beneficios para los representantes Fuente: Elaboración propia.

¿Qué actividad desarrollo usted, mientras su niño asiste al CDI?

Una cifra bastante significativa con respecto a las actividades que desarrollan los representantes entre ellos el $38.30 \%$ se dedica a la agricultura y ganadería. El $100 \%$ de usuario desarrolla alguna actividad productiva mientras su representado asiste al CDI.

El $93.62 \%$ de usuarios siente seguridad cuando su

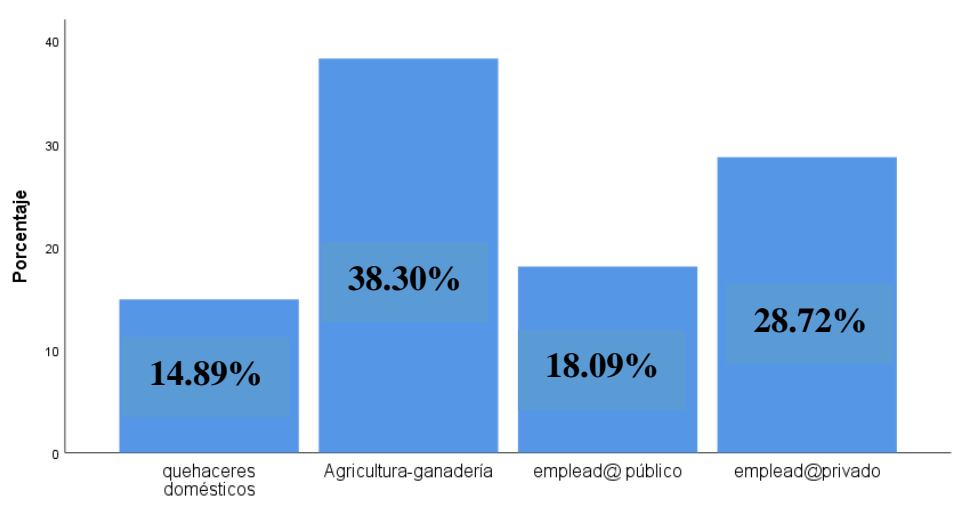

Figura 2. Actividades representantes CDI Fuente: Elaboración propia. 
Nivel de ingreso familiar

Los servicios que brinda el MIES conjuntamente con el GAD Municipal a través de los CDI, está enfocada en su mayoría a la población más vulnerable de cantón, es así que mediante la encuesta obtuvo datos alarmantes de ingresos por hogar, donde el $67.39 \%$ de usuarios ganan menos de $\$ 200.00$ dólares, su ingreso familiar no cubre ni el salario básico unificado (SBU), que en la actualidad es de $\$ 400.00$ para Ecuador.

Figura 3. Nivel de ingreso familiar

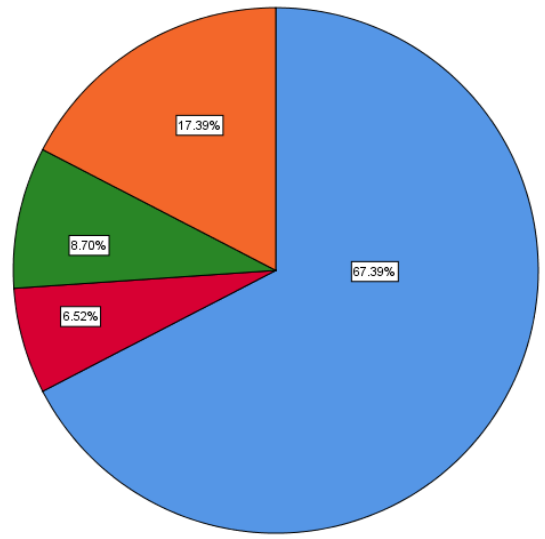

Fuente: Elaboración propia.

Problemas que enfrentan los hogares en la actualidad

Principalmente los representantes destacan como problemas que enfrentan los hogares son problemas económicos, desempleo, maltrato familiar, afecciones que se acentuaron a raíz de la pandemia COVID-19.

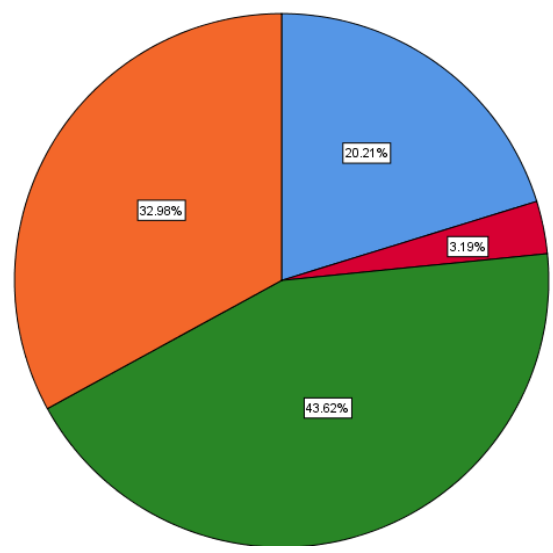

Figura 4. Problemas que enfrentan los hogares Fuente: Elaboración propia.

Para profundizar los aspectos de apoyo al desarrollo de los CDI se consideró importante levantar una entrevista a los funcionarios de la entidad que están involucradas con el desarrollo y funcionamiento de dichos centros, aplica las entrevistas a dos funcionarios tanto del GAD cantonal Chunchi y funcionaria del MIES.

El Ingeniero Jorge Luis Ortega en representación del Municipio de Chunchi comenta que como GAD municipal se han comprometido con la participación activa con las familias y la comunidad, dentro de las funciones que cumple el municipio es mejorar los resultados de los niños y niñas mediante atenciones integrales, promover el rol de mediación de la madre, padre, educador y familia para la adopción de las buenas prácticas de crianza, cuidado y 
protección mediante la consejería familiar. Dentro de los recursos económicos que el GAD Municipal asigna para el funcionamiento de los CDI, en el presupuesto es de \$66.616.37 dólares (2020), estos recursos están a destinados a: mantenimiento de los centros, energía eléctrica, agua potable, telecomunicaciones, trasporte, material de aseo, suministros de oficina, equipo de cómputo, nutricio, alimentación, desarrollo infantil, material didáctico, material fungible, la infraestructura, además personal técnico en las diferentes áreas tales como (jardinería, albañilería, plomería etc.)

El seguimiento que se da a los CDI, lo realizan mediante "La Unidad de Desarrollo", y "La Unidad de Desarrollo y Cultura" en la persona del Ing. Santiago Pérez, quien realiza monitoreo constante a los centros: inspecciones en cuanto a infraestructura, servicio de alimentación, atención de los niños por parte de las educadoras, inspección de necesidades básicas de los centros.

Los cambios que se esperaba en la comunidad con el servicio de los CDI es que la comunidad y los niños se beneficien de una adecuada estimulación temprana y alimentación en las edades correspondientes para garantizar un desarrollo óptimo en el futuro de los niños, considerando que ellos son el presente y el futuro de nuestro país, además que la provincia de Chimborazo es la que tiene mayor índice de desnutrición.

Los resultados críticos que el programa trata de lograr es subsanar y prever los índices de desnutrición y calidad de vida de los niños, como plantea el Sumak Kawsay.

El nivel de acogida que tienen los CDI en el cantón es excelente, debido a que el cuidado de los niños en el centro presenta grandes beneficios no solo en la alimentación y cuidado, también con los padres de familia ya que pueden trabajar con tranquilidad y no dejar a sus niños en casa solos.

Indudablemente se genera Desarrollo Local dice el vicealcalde, toda actividad que sea con fines o sin fines de lucro da resultados positivos, y más cuando es en ámbito social, debido a que los padres pueden laborar, y generan ellos también un trabajo para la ciudadanía, por eso creemos que este tipo de servicios brindan desarrollo no solo local también provincial, y así se dinamiza la economía.

Así culmina la participación de representante del GAD Municipal del Cantón Chunchi.

Por otra parte, la Ingeniera Paulina Moreano, funcionaria del MIES manifiesta: El MIES, a través de la Subsecretaria de Desarrollo Infantil Integral, ha emprendido un proceso de fortalecimiento de las políticas públicas de atención a la Primera Infancia mediante la intervención emblemática del Gobierno Nacional, a través del programa Misión Ternura (MT). La norma técnica vigente para la Modalidad MT - CDI, es la establecida mediante Acuerdo Ministerial 072 y tiene como objetivo regular y orientar la prestación del servicio de atención infantil y familiar en la modalidad (MT-CDI). 
La norma técnica como instrumento es de cumplimiento obligatorio para las unidades de atención MT - CDI del MIES - (atención directa y convenios).

Los CDI se promocionan por medio de focalizaciones en territorio, a través de líderes comunitarios y/o actores locales que son responsables de la promoción, vigilancia y garantía de los derechos de la mujer gestante y la niñez, además por los medios de comunicación oficiales del MIES.

El personal que labora en los CDI recibe capacitaciones permanentes. El MIES a través de la Formación Continua busca fortalecer las competencias, habilidades y destrezas del personal que trabaja en los servicios de atención que brinda el ministerio en sus distintas modalidades de atención.

La funcionaria destaca que para evaluar las metas alcanzadas se analizan indicadores como: Gobierno por Resultados (GPR), que es el instrumento informático nacional; módulo de aplicación de logros del SIIMIES (Sistema Integrado de Información del Ministerio de Inclusión Económica y Social, que permite mostrar los logros alcanzados en cuatro ámbitos de aprendizaje y desarrollo; aplicación del paquete integral de atenciones como lo establece el acuerdo interinstitucional 010 MIES-MSP, control de niño sano dos tomas marzo y octubre.

Dentro de los cambios que se esperaban en la comunidad como resultado de la entrega del programa de los CDI destaca los siguientes: que las familias y la comunidad se empoderen de los procesos para la vigilancia del cumplimiento de la RIA, la calidad de las atenciones por parte de las Instituciones Públicas como el MIES, Registro Civil, MSP, Educación; empoderamiento de derechos y obligaciones de las familias y la comunidad, para el cumplimiento de su rol en la crianza cuidado y protección de sus hijos e hijas; fomentar en las mujeres gestantes la realización de todos los controles de atención integral en salud y a las familias para controles de niño sano y esquema de vacunas; fomentar prácticas de higiene saludables: consumo de agua segura, lavado de manos y alimentación saludable; fomentar el consumo de lactancia materna; concientizar a las autoridades sobre la importancia de la nutrición y salud de su gente y todas las consecuencias de la malnutrición; mejorar la calidad de vida las personas que registran mayores niveles de vulnerabilidad y pobreza; niñas y niños con tendencia de crecimiento normal (Peso/Edad, Longitud/Edad, Talla/Edad); familias que reconozcan la importancia de un ambiente familiar protector con afecto y, confianza, que favorezca un entorno en el que los niños y niñas vivan sin riesgo o amenaza de vulneración de derechos.

Los resultados críticos que el programa trata de lograr es la reducción de la prevalencia de desnutrición crónica en las niñas y niños. 
Tomando con referencia lo planteado por Aguilar y por el Banco de Desarrollo de América Latina, se puede considerar en base a los resultados analizados que los centros de desarrollo infantil del cantón están cumpliendo con su acometido porque se están acogiendo a niños en estado de vulnerabilidad: con madres solteras en un $27.66 \%$, nivel de instrucción bajo (primaria) el $46.81 \%$, ruralidad, pobreza e ingresos bajos.

En base a los resultados obtenidos, se va a utilizar la fase de evaluación orientada por criterios propuesta por el Banco de Desarrollo.

\section{Fase de la evaluación orientada por criterios}

Aplicación

1. Construcción, reconstrucción identificación de la teoría

1.1. Resultados

1.2. Dimensión de procesos mecanismos de implementación.

1.3. Dimensión estructural: los recursos, organización $\quad \mathrm{y}$ otros elementos estructurales
Se realizó un análisis exhaustivo de las diferentes teorías que aborda este trabajo investigativo, como son: desarrollo local, política pública, centros de desarrollo infantil, MIES, Chunchi.

Los resultados obtenidos son positivos y cumplen con el objetivo para lo cual fueron creadas las políticas públicas, considerando el impacto que ha tenido el programa en la población.

Los Centros de Desarrollo Infantil, garantizan una alimentación saludable a las niñas y niños de acuerdo a la edad y el estado nutricional, de conformidad con las recomendaciones nutricionales diarias como se establece en el protocolo de externalización del servicio de alimentación y conforme a la normativa institucional, el reglamento para la Regulación y Control de Proveedores de Alimentos.

Identificación de la población de niñas y niños de uno a tres años, mujeres gestantes y familias de niñas y niños de 0 a 1 año de edad, a través de la búsqueda activa y el correspondiente operativo territorial.

Priorización de usuarias/os a ser atendidos por el CDI a partir del análisis de resultados de Vulnerabilidad y del Registro Social.

Iniciar las atenciones en los CDI en el marco de la protección integral, exigiendo que sean inclusivos e interculturales y aportando a los usuarios: atención receptiva, salud y nutrición, espacio de juego y aprendizaje, entornos protectores para una libre expresión de emociones.

Estrategia de liderazgo: Organizaciones gubernamentales y locales, quienes están en monitoreo continuo del funcionamiento de los CDI. Recursos humanos: Técnicos, educadoras, Supervisores, funcionarios de entidades contratantes GAD-MIES. Infraestructura, tecnología y finanzas: tres diferentes estructuras físicas ubicadas en tres localidades que son Chunchi, Gonzol y Llagos. Todas cuentan con aparatos tecnológicos y sus necesidades están cubiertas por las instituciones contratantes MIES-GAD municipal. Gestión del programa: Para que el programa se desarrolle debe existir población en condiciones de 
vulnerabilidad, para lo cual el GAD municipal acude al MIES para solicitar el funcionamiento del programa en la localidad. Mediante convenio se desarrolla este programa. Gestión del proceso: el GAD municipal es el ente encargado de focalizar ubicación, infraestructura, personal, alimentación etc., Los gastos del servicio son cubiertos por las dos entidades. Alianzas interinstitucionales: MIES - GAD Municipal

1.4. $\mathrm{La}$ perspectiva sistémica

2. Múltiples perspectivas sobre las teorías que subyacen a una intervención

2.1 Fuentes para obtener las pistas y sugerencias para construir la teoría.

3. Modelo lógico con perspectiva sistémica

4. Graficar la teoría

5. Determinación de los aspectos a evaluar

6. Métodos y técnicas

7. Análisis
Es muy importante el convenio que existe entre las instituciones del gobierno nacional y local, mismas que permiten el funcionamiento de estos CDI, los resultados obtenidos como producto del programa indican los grandes beneficios para los niños y sus familias. De no existir este servicio se evidenciarían altos índices de desnutrición, bajos ingresos en las familias, maltrato intrafamiliar, etc.

Considerando que muchas madres de familia no podían laborar, por no tener una persona a cargo de su hijo. Considerando los altos índices de desnutrición de los niños, por ser encargados o estar descuidados mientras sus padres laboran arduas jornadas. Conociendo que los niños y niñas tienen derechos establecidos en la Constitución, en los ODS etc. Se generó esta política pública de vital importancia para sus usuarios.

Realización de encuestas: a los usuarios de los servicios. Realización de entrevistas a actores claves: funcionaria CDI, funcionario GAD municipal. Revisión de documentación: misma que justifique la creación de políticas públicas. Observación de programas: prestando mayor atención a lo ejecutado que a lo planeado. Revisión profunda de la literatura: contemplada en esta investigación.

Organizar toda la información obtenida de las diferentes fuentes de consulta, como también a través de las encuestas y entrevistas. Resumir y clarificar el contenido de esta investigación. Redactar adecuadamente el documento objeto de estudio.

Después de depurar información y tener claro el objetivo de la investigación, desarrollar un documento que contenga lo más relevante e importante sobre el desarrollo Local y las políticas públicas en bienestar de los niños usuarios de los CDI.

Los aspectos a evaluar en esta investigación son los resultados, procesos y estructura. Que previamente la tenemos resuelta

Los métodos y técnicas utilizados para esta investigación están detallados anteriormente.

La verificación de funcionamiento del programa es favorable ya que, por medio de la investigación y aplicación de encuestas y entrevistas, pudimos evidenciar que cumplen los objetivos para lo que fueron creados los CDI.

\section{Tabla 3: Resultado de la evaluación en territorio Fuente: Elaboración propia.}




\section{Evaluación}

Logros en territorio

De eficacia Los CDI están siendo ocupados mayoritariamente por personas en estado de vulnerabilidad, pobreza y pobreza extrema.

De metas Los CDI, cumplen con los cupos asignados para cada uno de ellos. El nivel de cobertura está completo.

De eficiencia

Los usuarios reciben un beneficio de carácter social, son personas en estado de vulnerabilidad y pobreza quienes a graves de estos centros reciben apoyo integral,

de efectos

de impacto

De satisfacción
Los resultados han sido satisfactorios ya que familias se han visto beneficiadas, generando ingresos para sus hogares, y los niños reduciendo niveles de desnutrición.

Se genera desarrollo Local en los sectores donde funcionan los CDI, ya que las familias laboran, se reduce índices de violencia intrafamiliar y se reduce índices de desnutrición.

El grado en que la población valora la existencia de la política pública con respecto al funcionamiento de los CDI es satisfactoria ya que no trae un solo beneficio en respecto al usuario sino el beneficio es global para la familia y la sociedad.

Tabla 4: Aplicación de las etapas de evaluación orientada por criterios

Fuente: Elaboración propia.

\section{Conclusiones.}

Al finalizar este trabajo investigativo, mismo que fue desarrollado con la mayor dedicación, ética y profesionalismo se llegó a las siguientes conclusiones:

- El desarrollo Local siendo un proceso de trasformación de la sociedad busca el bienestar de la colectividad, mediante la creación de políticas públicas, las mismas que van encaminadas a grupos de atención prioritaria. En el Ecuador, una de las políticas públicas con mayor relevancia e importancia es velar por los niños, por este motivo el Gobierno Nacional ha creado centros de desarrollo infantil, cuyo objetivo es la protección de niños en su primera infancia a nivel nacional. Considerando que el desarrollo infantil es fundamental para alcanzar las metas de desarrollo sostenible y la garantía de los derechos durante los primeros años de vida.

- La política pública creada en beneficio de niños y niñas en su primera infancia ha incidido de manera positiva en la población. El aporte que este servicio brinda a la localidad es valioso, están destinados a poblaciones en estado de vulnerabilidad, pobreza y pobreza extrema. Los niños presentan mayor seguridad, desempeño y confianza a raíz que acuden a los CDI. Mediante una alimentación sana se logró 
reducir índices de desnutrición. Mediante los talleres que el MIES brinda a los padres de manos y alimentación saludable en sus hogares. El 100\% de las familias usuarias de los servicios que brindan los Centros de Desarrollo Infantil sienten bienestar al dejar a sus niños en estas instituciones Se logra mayor incorporación de madres a la vida laboral, generando mayores ingresos para su hogar e incidiendo en la calidad de vida para sus familias.

- Evidentemente la política pública sobre los funcionamientos de los CDI, ha tenido un gran impacto social en los usuarios. Los niños usuarios de los CDI presentan avances en cuanto a su desarrollo fisico, psicologico, emocional y psicomotriz. Uno de los problemas que el programa trata de mitigar es la desnutricion en niños en edades de 1 a 3 años, misma que a traves una alimentacuion balanceada y la ingesta de cuatro comidas diarias se va logrando controlar. Se genera Desarrollo local, mediante la prestación de este servicio, los niños que son parte de estos programas se encentran en mejores condiciones de alimentación, cuidados, estimulación temprana, etc. Los padres de familia pueden laborar, generando mayores ingresos para sus hogares. Se reduce la violencia de género. Es fundamental mantener este tipo de políticas públicas mismas que están enfocadas en la población más vulnerable del cantón, en ese caso los niños que representan el presente y el futuro de la población. Todo va articulado de manera positiva gracias a la aplicación de políticas públicas de este tipo.

\section{Referencias Bibliográficas.}

Aguilar Astorga, C. R. (2017). Evaluación de políticas públicas, Una aproximación.

Araujo, M. C., \& López-Boo, F. (2015). Los servicios de cuidado infantil en América Latina y el caribe. Trimestre Económico, 82(326), 249-275. https://doi.org/10.20430/ete.v82i326.165

Asamble Nacional. (2008). Constitución de la República del Ecuador 2008. https://doi.org/10.2307/j.ctvm204k6.6

Banco de Desarrollo de América Latina. (2016). La evaluación pragmática de las políticas públicas. In La evaluación de políticas. Fundamentos conceptuales y analíticos. http://scioteca.caf.com/handle/123456789/1008

Banks, G. (2009). Evidence-based policy making: What is it? How do we get it? (ANU Public Lecture Series, presented by ANZSOG, 4 February), Productivity Commission, Canberra. (Issue February).

CEPAL. (2018). La Ineficiencia de la Desigualdad. La Ineficiencia de La Desigualdad. https://doi.org/10.18356/ff8b886e-es 
Charry, G. P., \& Pérez, J. E. A. (2014). Las políticas públicas de desarrollo empresarial e innovación desde la perspectiva de la nueva gestión pública y la gobernanza: caso CREAME. Apuntes Del Cenes, 32(56), 175. https://doi.org/10.19053/22565779.2447

Codigo de la Niñez y Adolescencia. (2013). Codigo de la Niñez y Adolescencia. Codigo de La Niñez y Adolescencia, O(2002), 1-45. https://doi.org/10.1111/j.10958312.1989.tb01569.x

Contreras, J. I., Rojas, V., \& Contreras, L. (2015). Análisis de programas relacionados con la intervención en niños, niñas y adolescentes vulnerados en sus derechos: La realidad chilena. Psicoperspectivas, 14(1), 89-102. https://doi.org/10.5027/PSICOPERSPECTIVAS-VOL14-ISSUE1-FULLTEXT-528

COOTAD. (2010). Código Orgánico Organización Territorial Autonomía Descentralización. 174. http://www.oas.org/juridico/pdfs/mesicic4_ecu_org.pdf\%0Ahttp://www.ame.gob.ec/a $\mathrm{me} / \mathrm{pdf} /$ cootad_2012.pdf

Díaz Jesús. (2018). Principios Básicos del Desarrollo Local. 11-31. https://www.faeditorial.es/capitulos/agente-de-desarrollo-y-empleo-local.pdf

European Environment Agency (EEA). (2019). Orientaciones para la evaluación de planes y políticas públicas para la primera infancia. 53(9), 1689-1699. https://doi.org/10.1017/CBO9781107415324.004

Falconí Benítez, F. (2017). Solidaridad sostenible La codicia es indeseable. https://biblio.flacsoandes.edu.ec/libros/digital/57015.pdf

GAD Municipal Chunchi. (2014). PDOT Chunchi.

Gallicchio, E. (2017). Desarrollo local y cooperación al desarrollo: ¿una nueva generación de plataformas de cooperación para el desarrollo local?. 63-73. https://doi.org/10.29192/claeh.36.1.3

Guardamagna, M., \& Reyes, M. (2019). El desafío de la implementación de políticas públicas participativas para el desarrollo del territorio The challenge of implementing participation in public policies regarding territorial development. 18(59), 1003-1033. https://doi.org/10.22136/est20191284

Hombrados Mendieta, M., \& Olmos Ruiz, C. (2016). Apoyo social, salud mental y situación económica en mujeres de familias monoparentales y biparentales usuarias de los Servicios Sociales. 5(5), 5-15.

INEC Instituto Nacional de Estadísticas y censos. (2019). Encuesta nacional de relaciones familiares $y$ violencia de genero. 17-19. 
http://www.ecuadorencifras.gob.ec/documentos/web-

inec/Estadisticas_Sociales/sitio_violencia/presentacion.pdf

INEC. (14 de octubre de 2020). Ecuador en cifras. Obtenido de https://www.ecuadorencifras.gob.ec/estadisticas/

INEC Instituto Nacional de Estadísticas y censos. (2020). Encuesta Nacional de Salud y Nutrición. Ensanut, 1, 47. https://doi.org/10.1017/CBO9781107415324.004

Mauricio, L., Gonz, C., Lira, I. S., Jim, E. M., Mauricio, L., Gonz, C., Jim, E. M., Silva Lira, I., Jim, E. M., Mauricio, L., \& González, C. (2003). Planificación estratégica territorial y politicas publicas para el desarrollo local.

Méndez Coto, M. (2016). Multilateralismo, gobernanza y hegemonía en la estructura económica internacional: del G7 al G20. Relaciones Internacionales: Revista Académica Cuatrimestral de Publicación Electrónica, 0(31), 13-32.

Ministerio de Inclusión Económica y Social. (2013). Desarrollo Infantil Integral. Política Publica, $\quad 120 . \quad$ http://www.inclusion.gob.ec/wpcontent/uploads/downloads/2013/11/Libro-de-Políticas-Públicas.pdf

Ministerio de Inclusión Económica y Social. (2019a). Norma técnica, Misión ternura-CDI (1st ed.). Editogran-Medios Públicos EP. https://www.inclusion.gob.ec/wpcontent/uploads/2020/01/Norma-Técnica-MT-CDI_compressed.pdf

Ministerio de Inclusión Económica y Social. (2019b). NORMA TÉCNICA MISIÓN TERNURA MODALIDAD CENTROS DE DESARROLLO INFANTIL - CDI ACUERDO MINISTERIAL 072 DIRECCIÓN DE SERVICIOS DE CENTROS DE.

Muller, P. (2010). Las políticas públicas - Tercera Edición.

Naciones Unidas. (2016). Declaración de Quito sobre Ciudades y Asentamientos Humanos Sostenibles para Todos. 1-33.

Naciones Unidas/CEPAL. (2019). La Agenda 2030 y los Objetivos de Desarrollo Sostenible: una oportunidad para América Latina y el Caribe. Objetivos, metas e indicadores mundiales. In Publicación de las Naciones Unidas.

ONU. (2015). Transformar nuestro mundo: la Agenda 2030 para el Desarrollo Sostenible. Asamblea

General, 15900 ,

40. http://www.un.org/ga/search/view_doc.asp?symbol=A/70/L.1\&Lang=S

Pino Montoya, J. W. (2017). Aspectos metodológicos para evaluar una política pública. RHSRevista Humanismo y Sociedad, 5(1), 1-7. https://doi.org/10.22209/rhs.v5n1a01 
SENPLADES. (2017). Plan Nacional de Desarrollo 2017-2021. Toda una Vida. 84. http://www.planificacion.gob.ec/wp-content/uploads/downloads/2017/10/PNBV-26OCT-FINAL_0K.compressed1.pdf

Secretaría Técnica Planifica Ecuador. (2019). Lineamientos para articulación entre el pdot con la agenda 2030 y los ods. 4-36.

SNI. (10 de septimbre de 2020). Secretaria Técnica Planifica eEuador. Obtenido de Sistema Nacional de Información: https://sni.gob.ec

SIISE. (14 de Octubre de 2020). Secretaria Técnica Plan Todo una Vida. Obtenido de SIISE: https://www.todaunavida.gob.ec/sistema-integrado-de-indicadores-sociales-delecuador-siise-2/

UNICEF. (2020). Impacto del COVID-19 en los niños, niñas, adolescentes y sus familias en América Latina y el Caribe. 1-6.

Urbanos, R. (2012). Evaluación de políticas públicas. 1-30.

Villaroel Ojeda, A., Castells Carrión, P., \& Castro, A. (2018). Evaluación de programas y políticas públicas en Ecuador: oportunidades y desafíos. 10, 43-74.

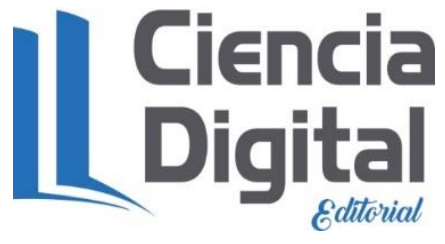




\section{PARA CITAR EL ARTÍCULO INDEXADO.}

Quezada Vintimilla , V. P., Rosales Namicela , M. B., \& Castillo Ortega , Y. (2021). Análisis del impacto de la política de atención a niños de 1 a 3 años, en el bienestar de sus familias en el caso del cantón Chunchi. Ciencia Digital, 5(1), 133-157. https://doi.org/10.33262/cienciadigital.v5i1.1520

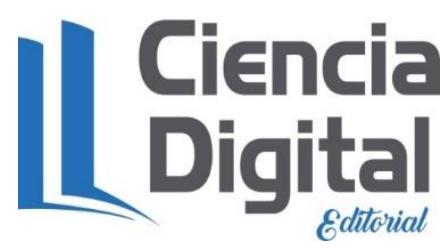

El artículo que se publica es de exclusiva responsabilidad de los autores y no necesariamente reflejan el pensamiento de la Revista Ciencia Digital.

El artículo queda en propiedad de la revista y, por tanto, su publicación parcial y/o total en otro medio tiene que ser autorizado por el director de la Revista Ciencia Digital.
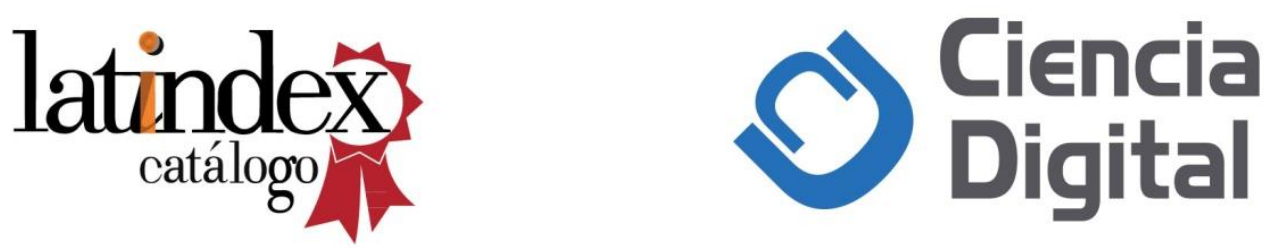